\title{
Does the Side Matter? A Retrospective Cohort Study Comparing Left and Right Pure Laparoscopic Donor Nephrectomies
}

\author{
Philip Zeuschner $^{a} \quad$ Michael Stöckle $^{a}$ Robert Peters $^{b}$ Kurt Miller $^{b}$ \\ Lutz Liefeldt $^{c}$ Fabian Halleck ${ }^{c}$ Klemens Budde ${ }^{c}$ Linda Hennig ${ }^{c}$ \\ Frank Friedersdorffc, d

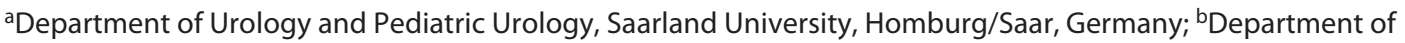 \\ Urology, Charité-Universitätsmedizin Berlin, Corporate Member of Freie Universität Berlin, Humbold-Universität zu \\ Berlin, Berlin Institute of Health, Berlin, Germany; 'Department of Nephrology, Charité-Universitätsmedizin Berlin, \\ Corporate Member of Freie Universität Berlin, Humbold-Universität zu Berlin, Berlin Institute of Health, Berlin,

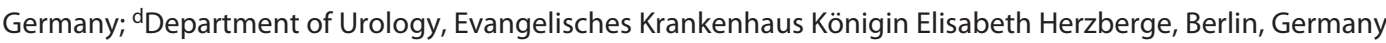

\section{Keywords}

Living kidney donation · Laparoscopic donor nephrectomy · Minimally invasive surgery

\section{Abstract \\ Objectives: Right laparoscopic donor nephrectomy (RLDN) is no longer regarded inferior to left LDN (LLDN). However, this knowledge is based on many studies suffering from in- herent learning curves, center-specific imbalances, and dif- ferent laparoscopic techniques. Methods: Pure LDNs at a high-volume referral center from 2011 to 2016 were retro- spectively analyzed. Patient, graft characteristics, outcomes of LDNs, and corresponding open kidney transplantations were compared between LLDN and RLDN including a follow- up. Results: 160 (78.4\%) LLDNs and 44 (21.6\%) RLDNs only differed regarding graft characteristics, as more right grafts had multiple veins ( 34.1 vs. $6.9 \%, p<0.001)$ and worse scin- tigraphic function (44 vs. $51 \%, p<0.001$ ). RLDNs were short- er (201 vs. $220 \mathrm{~min}, p=0.032$ ) with longer warm ischemia time (165 vs. $140 \mathrm{~s}, p<0.001)$, but left grafts were transplant- ed faster (160 vs. $171 \mathrm{~min}, p=0.048$ ). Recipients of right kid- neys had more postoperative complications (grade 3: 25.6}

vs. $11.3 \%, p=0.020$ ). At a follow-up of 45 (range 6-79) months, neither the kidney function, nor death-censored graft (5-year: LLDN 89 vs. $92 \%, p=0.969)$ and patient survival (5-year: LLDN 95 vs. $98 \%, p=0.747$ ) differed. Conclusions: Pure LLDN and RLDN can have different outcomes at high-volume centers, especially higher complications for recipients of right grafts. However, long-term function and graft survival are the same irrespective of the chosen side.

(c) 2021 The Author(s)

Published by S. Karger AG, Basel

\section{Introduction}

Laparoscopic donor nephrectomy (LDN) still represents the standard surgical approach for living kidney donation, even 25 years after the first successful report [1]. However, its introduction initially confronted transplant surgeons with significant challenges due to the highly variable vessel anatomy and anatomical differences between left and right grafts [2]. Hence, most transplant

L.H. and F.F. contributed equally. karger@karger.com www.karger.com/uin

Karger $\stackrel{\text { ' }}{5}$ BOPEN ACCESS
(C) 2021 The Author(s)

Published by S. Karger AG, Basel

This is an Open Access article licensed under the Creative Commons Attribution-NonCommercial-4.0 International License (CC BY-NC) (http://www.karger.com/Services/OpenAccessLicense), applicable to the online version of the article only. Usage and distribution for commercial purposes requires written permission. 
centers perform far more left than right LDNs, as leftsided grafts usually have a longer renal vein, and right LDN (RLDN) is only conducted ifleft-sided LDN (LLDN) appears unfavorable [3-6]. These factors contribute to a lower expertise in RLDN for most transplant surgeons.

During the first years after the introduction of LDN, this lack of experience caused more vascular complications and graft thromboses after RLDN and some authors described vascular complication rates of $40 \%$ after RLDN $[3,7]$. Nowadays, optimized techniques for RLDN enable the transplant surgeons to preserve a sufficient length of the renal vein, yielding better results [8]. For this reason, RLDN is no longer considered to be inferior to LLDN [1, $4,7]$.

Regardless of this, many analyses do not differentiate between the highly diverse minimally invasive techniques. Moreover, most studies are impacted by inherent learning curves of the transplant surgeons $[7,9,10]$. Hence, the potential impact of the side of LDN on outcomes could be underestimated. For this reason, we performed a large single-center comparison of pure laparoscopic donor nephrectomies comparing RLDN with LLDN from 2011 to 2016, including the donor and recipient outcomes and a follow-up. As we have been performing LDNs since 1999, the effect of learning is negligible within this cohort [11]. To compare and assess the impact of the side of LDN and other factors on complications which defined the primary outcome, multiple regression analyses were conducted.

\section{Materials and Methods}

All living kidney donations among adults at one tertiary referral center (Charité Berlin) between 2011 and 2016 were retrospectively analyzed. LDN was performed with a pure laparoscopic technique as described previously $[12,13]$. In brief, 4 ports were used, the surgery was conducted without hand-assistance and the kidney was extracted through an enlarged lateral trocar incision measuring 5-6 cm [14]. All kidney transplantations (KTs) were conducted in an open extraperitoneal approach to the iliac fossa. All donors underwent preoperative imaging by computed tomography or magnetic resonance angiography and renal scintigraphy. The graft's side was chosen on a case-by-case decision, based on the proportion of split-renal function and the vascular anatomy [15].

The donor and recipient's characteristics were obtained and the graft's side, split-renal function, and number of arteries and veins served as organ-specific factors. The intraoperative (operating time, warm ischemia time [WIT], cold ischemia time [CIT], anastomosis time, and complications) and postoperative results (complications according to Clavien-Dindo within 30 days after surgery, length of stay) were compared between RLDN and LLDN and the corresponding open KTs. The delayed graft function (DGF) rates,

Comparison of Right versus Left

Laparoscopic Donor Nephrectomies defined as the need for dialysis within 1 week after transplantation without dialysis to deplete potassium only were obtained. The kidney function of the donor was compared before $\mathrm{LDN}$ with the function at discharge. For the recipients, a follow-up was conducted regarding the kidney function, patient, and death-censored graft survival. To assess the impact of the side of LDN, but also donor-, graft- and recipient-specific factors on postoperative complications for the donor and recipient, multiple binary logistic regression analyses were performed. The intra- and postoperative complications of the donors and recipients were defined as the primary outcome. As secondary outcome, the kidney function of the donor and graft function of the recipient were compared.

The statistical analysis of the data was conducted using IBM SPSS Statistics Version 25 (IBM Corp., Armonk, USA). Categorical variables were reported as frequencies and proportions, continuous data as the median and range. Fisher's exact, Mann-Whitney U, Kruskal-Wallis, and Wilcoxon tests were applied. The death-censored graft and patient survival were analyzed with Kaplan-Meier method and log-rank tests. All tests were two-sided, and $p$ values $<0.05$ were considered significant. This retrospective study has been performed according to the Declaration of Helsinki. Approval by the Institutional Review Board of Charité Hospital Berlin was not necessary, and the need for informed consent was waived because of this study's retrospective, noninterventional design and because patient data confidentiality and privacy were maintained.

\section{Results}

\section{Donor, Recipient Demographics, and Graft}

\section{Characteristics}

Overall, 205 LDNs were included with 160 (78.4\%) left and $44(21.6 \%)$ right LDNs, one case had to be excluded due to unknown side (Fig. 1). The median caseload of 34 (range 20-48) LDNs per year was significantly higher for LLDNs (26/year [range 18-38] vs. 8/year [2-10], $p=$ 0.014). 150 left kidneys were transplanted to adults, whereof 133 (88.7\%) were assigned to the contralateral iliac fossa. As for right kidneys, 30 (69.8\%) were transplanted to the contralateral iliac fossa. All interventions were performed by 7 experienced laparoscopic surgeons with a mean caseload of 29 LDNs.

The donor characteristics did not significantly differ between LLDN and RLDN (Table 1). More left grafts had multiple arteries (21.3 vs. $9.1 \%$, n.s.), but right grafts had multiple veins significantly more often ( 34.1 vs. $6.9 \%, p<$ 0.0019 ) and a worse scintigraphic function (44 vs. $51 \%$, $p<0.001)$.

The recipients were 46 years old and mostly male. Autosomal-dominant polycystic kidney disease (LLDN 14.7 vs. RLDN 18.6\%) and IgA nephropathy (16 vs. 14\%) were the most frequent underlying diseases (Table 1). In both groups, nearly $50 \%$ of recipients had a history of smoking 
Table 1. Donor, graft, and recipient characteristics, compared between LLDN and RLDN

\begin{tabular}{|c|c|c|c|}
\hline & LLDN & RLDN & $p$ value \\
\hline Donor & $n=160$ & $n=44$ & \\
\hline Age, years & $51(21 ; 78)$ & $51.5(28 ; 73)$ & 0.902 \\
\hline Male gender & $63(39.4 \%)$ & $11(25 \%)$ & 0.110 \\
\hline BMI, $\mathrm{kg} / \mathrm{m}^{2}$ & $25.9(17.6 ; 35.6)$ & $25.4(18.1 ; 36.1)$ & 0.843 \\
\hline Hypertension & $36(22.5 \%)$ & $8(18.2 \%)$ & 0.680 \\
\hline Diabetes & $2(1.3 \%)$ & $1(2.3 \%)$ & 0.519 \\
\hline History of smoking & $86(53.8 \%)$ & $25(56.8 \%)$ & 0.733 \\
\hline Graft & $n=160$ & $n=44$ & \\
\hline Multiple arteries & $34(21.3 \%)$ & $4(9.1 \%)$ & 0.081 \\
\hline Multiple veins & $11(6.9 \%)$ & $15(34.1 \%)$ & $<0.001$ \\
\hline DPTA-clearance, $\mathrm{mL} / \mathrm{min} / \mathrm{m}^{2}$ & $121(76 ; 235)$ & $131(82 ; 237)$ & 0.137 \\
\hline Scintigraphic function & $51 \%(39 \% ; 58 \%)$ & $44 \%(38 \% ; 57 \%)$ & $<0.001$ \\
\hline Recipient & $n=150$ & $n=43$ & \\
\hline Age, years & $46(18 ; 72)$ & $47(18 ; 76)$ & 0.807 \\
\hline Male gender & $110(73.3 \%)$ & $26(60.5 \%)$ & 0.139 \\
\hline $\mathrm{BMI}, \mathrm{kg} / \mathrm{m}^{2}$ & $24.7(17.1 ; 40.8)$ & $24.7(16.8 ; 37.8)$ & 0.969 \\
\hline Underlying disease & & & 0.713 \\
\hline ADPKD & $22(14.7 \%)$ & $8(18.6 \%)$ & 0.478 \\
\hline IgA nephropathy & $24(16 \%)$ & $6(14 \%)$ & 1.0 \\
\hline FSGS & $19(12.7 \%)$ & $4(9.3 \%)$ & 0.789 \\
\hline Other & $84(56 \%)$ & $18(41.9 \%)$ & 1.0 \\
\hline Hypertension & $139(92.7 \%)$ & $40(93 \%)$ & 1.0 \\
\hline History of smoking & $76(50.7 \%)$ & $18(41.9 \%)$ & 0.387 \\
\hline Preemptive KTs & $46(30.7 \%)$ & $12(27.9 \%)$ & 0.851 \\
\hline Waiting time, days & $210.5(0 ; 3,456)$ & $205(0 ; 2,882)$ & 0.650 \\
\hline$\geq 1$ prior transplantation & $7(4.7 \%)$ & $1(2.3 \%)$ & 0.687 \\
\hline
\end{tabular}

Fig. 1. Flowchart illustrating the number

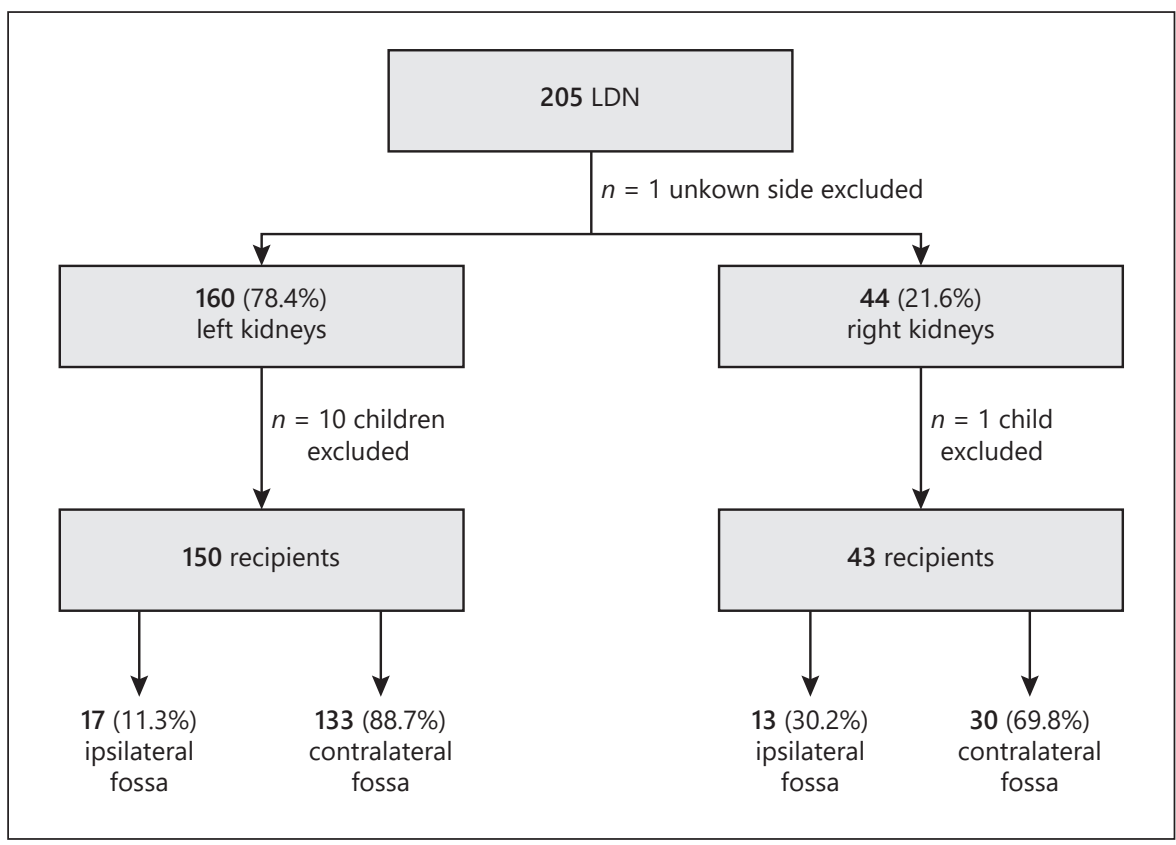
and distribution of grafts. 
Table 2. Surgical outcomes of LDNs

\begin{tabular}{lccc}
\hline & LLDN $(n=160)$ & RLDN $(n=44)$ & $p$ value \\
\hline Intraoperative & & & \\
Operating time, min & $220(120 ; 392)$ & $201(152 ; 285)$ & 0.032 \\
WIT, s & $140(32 ; 300)$ & $165(25 ; 310)$ & 0.001 \\
Complications & $5(3.1 \%)$ & $3(6.8 \%)$ & 0.761 \\
Postoperative & & & 1.0 \\
Complications & $128(80.0 \%)$ & $36(81.8 \%)$ & 0.582 \\
Grade 0 & $16(10 \%)$ & $6(13.6 \%)$ & 0.532 \\
Grade 1 & $13(8.1 \%)$ & $2(4.5 \%)$ & 1.0 \\
Grade 2 & $2(1 \%)$ & - & 1.0 \\
Grade 3a & $1(0.5 \%)$ & - & 1.0 \\
Grade 3b & - & $5(3 ; 9)$ & 0.297 \\
Grade $>3$ & $5(3 ; 18)$ & & \\
Length of stay, days & & & \\
\hline
\end{tabular}

Data is given as absolute (relative) frequency or median (range). LLDN, left laparoscopic donor nephrectomy; RLDN, right laparoscopic donor nephrectomy; WIT, warm ischemia time.

Table 3. Surgical outcomes of open KTs after LLDN and RLDN including the follow-up

\begin{tabular}{|c|c|c|c|}
\hline & $\operatorname{LLDN}(n=150)$ & RLDN $(n=43)$ & $p$ value \\
\hline \multicolumn{4}{|l|}{ Intraoperative } \\
\hline Operating time, $\min$ & $160(96 ; 362)$ & $171(120 ; 393)$ & 0.048 \\
\hline CIT, s & $141.5(38 ; 238)$ & $154.5(30 ; 218)$ & 0.086 \\
\hline Anastomosis time, $\min$ & $38.5(26 ; 71)$ & $40(30 ; 70)$ & 0.051 \\
\hline Complications & $10(6.7 \%)$ & $3(7 \%)$ & 1.0 \\
\hline \multicolumn{4}{|l|}{ Postoperative } \\
\hline Complications & & & 0.01 \\
\hline Grade 0 & $104(69.3 \%)$ & $21(48.8 \%)$ & 0.013 \\
\hline Grade 1 & $4(2.7 \%)$ & $4(9.3 \%)$ & 0.075 \\
\hline Grade 2 & $25(16.7 \%)$ & $7(16.3 \%)$ & 0.952 \\
\hline Grade 3a & $2(1.3 \%)$ & - & 1.0 \\
\hline Grade $3 b$ & $15(10.0 \%)$ & $11(25.6 \%)$ & 0.020 \\
\hline Grade $>3$ & - & - & 1.0 \\
\hline Length of stay, days & $12(6 ; 37)$ & $13(7 ; 34)$ & 0.065 \\
\hline \multicolumn{4}{|l|}{ Graft function } \\
\hline DGF & $8(5.3 \%)$ & $5(11.6 \%)$ & 0.169 \\
\hline \multicolumn{4}{|l|}{ Follow-up } \\
\hline \multicolumn{4}{|l|}{ Patient survival } \\
\hline 1 year & $99 \%$ & $100 \%$ & 0.747 \\
\hline 3 years & $97 \%$ & $98 \%$ & \\
\hline 5 years & $95 \%$ & $98 \%$ & \\
\hline \multicolumn{4}{|c|}{ Death-censored graft survival } \\
\hline 1 year & $100 \%$ & $100 \%$ & 0.969 \\
\hline 3 years & $96 \%$ & $92 \%$ & \\
\hline 5 years & $89 \%$ & $92 \%$ & \\
\hline
\end{tabular}

Data are given as absolute (relative) frequency or median (range). CIT, cold ischemia time; DGF, delayed graft function; LLDN, left laparoscopic donor nephrectomy; RLDN, right laparoscopic donor nephrectomy; KT, kidney transplantation. 
Table 4. Multiple regression analysis to predict postoperative complications for the donor (left) and for the recipient (right) after LDN

\begin{tabular}{|c|c|c|c|c|}
\hline \multirow[t]{2}{*}{ Variable } & \multicolumn{2}{|l|}{ Donor } & \multicolumn{2}{|l|}{ Recipient } \\
\hline & OR (95\% CI) & $p$ value & OR (95\% CI) & $p$ value \\
\hline \multicolumn{5}{|l|}{ LDN side } \\
\hline LLDN & - & 0.788 & $0.4(0.2 ; 0.79)$ & 0.009 \\
\hline \multicolumn{5}{|l|}{ Donor } \\
\hline Gender & - & 0.200 & - & 0.811 \\
\hline Age, years & - & 0.440 & - & 0.324 \\
\hline \multicolumn{5}{|l|}{ Graft } \\
\hline Multiple vessels & - & 0.450 & - & 0.723 \\
\hline Retroaortic left vein & - & 0.311 & - & 0.294 \\
\hline \multicolumn{5}{|l|}{ Recipient } \\
\hline Prior transplantations & & & - & 0.326 \\
\hline Left iliac fossa & & & - & 0.558 \\
\hline Waiting time, days & & & - & 0.123 \\
\hline
\end{tabular}

95\% CI, 95\% confidence interval; LLDN, left laparoscopic donor nephrectomy; OR, odds ratio; RLDN, right laparoscopic donor nephrectomy.

and for $>95 \%$ of kidney recipients, it was their first KT (95.3 vs. 97.7\%). AB0-compatibility and HLA-mismatches were no differences between the groups (online suppl. Table S1; for all online suppl. material, see www. karger.com/doi/10.1159/000517882).

\section{Surgical Outcomes}

Left LDNs took 19 min longer than RLDNs (220 vs. $201 \mathrm{~min}, p=0.032$ ), but had a shorter WIT (140 vs. $165 \mathrm{~s}$, $p<0.001$, Table 2). The intraoperative complication rate was comparable (LLDN 3.1 vs. $6.8 \%, p=0.373$ ). During $2(1.3 \%)$ LLDNs, the spleen was accidentally injured, 1 $(0.6 \%)$ left graft was damaged during kidney removal with a tear of the capsula that could be sutured, another left renal vein had to be shortened as it was torn and once a superficial cut within the mesocolon had to be sutured. Regarding the RLDNs, 2 (4.5\%) cases had to be converted to open surgery due to bleedings and one $(2.2 \%)$ ureteropelvic junction turned out as obstructed. Of note, no LLDN had to be converted. The postoperative complication rates did not differ. Only LLDNs were followed by 3 (1.9\%) major complications with one bronchoscopy due to dyspnea, one retention of chylous ascites which had to be punctured and one bleeding from the abdominal internal oblique muscle which had to be treated surgically. The kidney donors were discharged after 5 days (range 3; 18 days) in both groups.

For the corresponding KTs, the operating times were longer for right grafts (171 vs. $160 \mathrm{~min}, p=0.048$, Ta- ble 3). Bleedings were the most common intraoperative complication and occurred during 3 (7\%) KTs of right kidneys and 4 (2.7\%) KTs of left kidneys. During 4 (2.7\%) KTs of left kidneys, the anastomosis had to be sutured again, one $(0.7 \%)$ graft had perfusion deficits and once the renal parenchyma was accidently injured. Recipients of right grafts had more postoperative complications of higher grades (Table 3). 21 (48.8\%) recipients of right grafts had a complication-free course, in contrast to 104 (69.3\%) recipients of left kidneys ( $p=0.013)$. Postoperative major complications occurred twice as often after transplantations of right grafts (25.6 vs. $11.3 \%, p=0.020)$. For right grafts, $4(9.3 \%)$ vascular problems (2 arterial kinkings, one arterial stenosis, one venous malperfusion) and $3(6.8 \%)$ lymphoceles were the most common postoperative complications. For left grafts, 5 (3.1\%) wound dehiscence and $2(1.3 \%)$ lymphoceles were most frequent (online suppl. Table S2). The DGF rates were higher for right grafts, but this difference was not statistically significant (RLDN 11.6 vs. $5.3 \%, p=0.169$ ). When applying a wider DGF definition including also dialysis to deplete potassium only, the DGF rates neither differed (RLDN 11.6 vs. $6 \%, p=0.210$ ).

In the multiple regression analyses, neither the donor, recipient nor graft characteristics impacted the postoperative complication rates (Table 4). Only the side of LDN was associated with postoperative complications of the recipient (odds ratio $0.4, p=0.009$ ), but not with complications of the donor. 
Fig. 2. Kidney function of the donor (left) and graft function (right) during followup, compared between LLDN and RLDN. ${ }^{*} p<0.05,{ }^{* *} p<0.01,{ }^{* * *} p<0.001$. RLDN, right laparoscopic donor nephrectomy; LLDN, left laparoscopic donor nephrectomy.
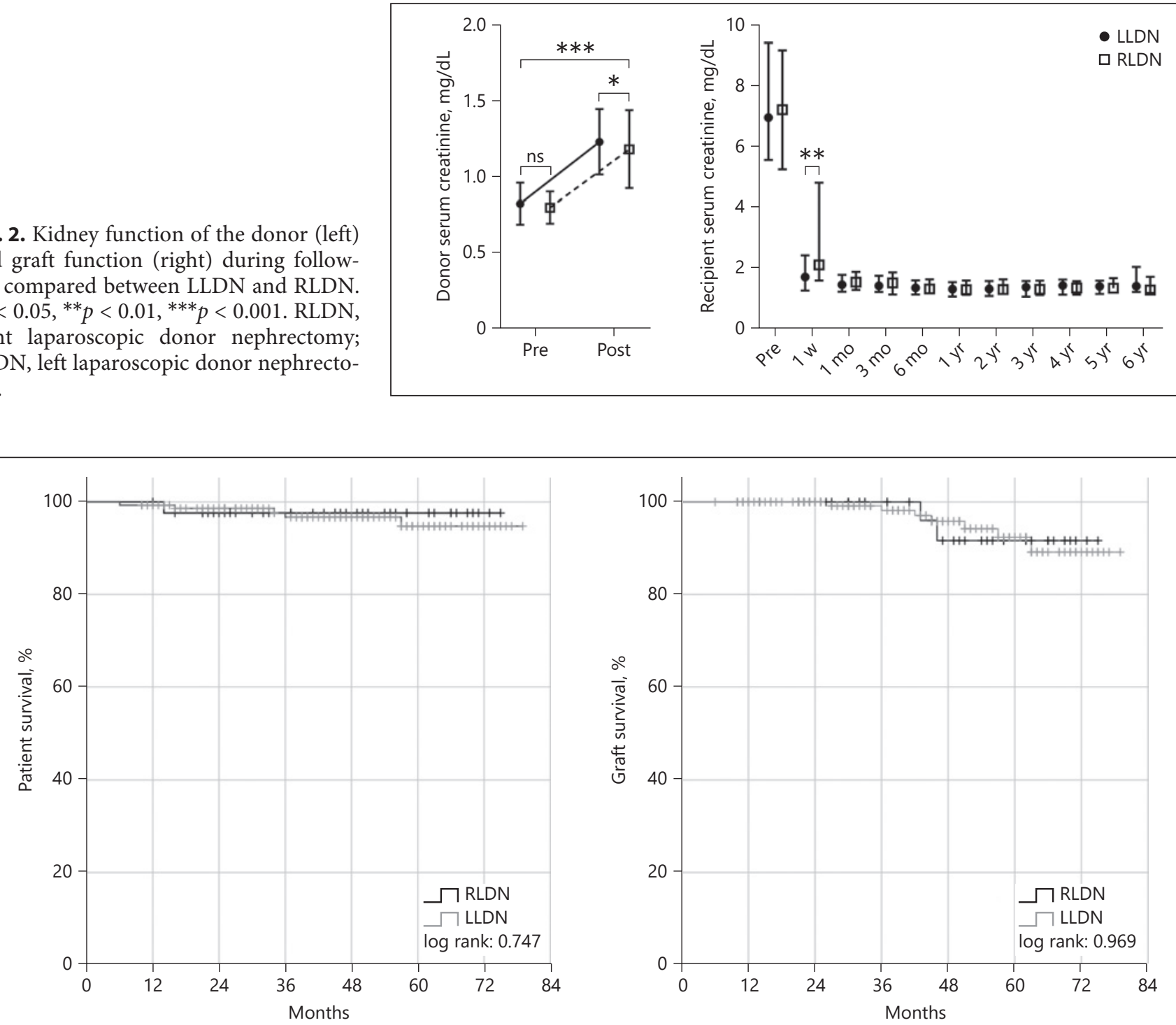

Fig. 3. Patient and death-censored graft survival during follow-up, stratified by kidney side (grey: LLDN and black: RLDN). LLDN, left laparoscopic donor nephrectomy; RLDN, right laparoscopic donor nephrectomy.

\section{Follow-Up}

For all donors, the serum creatinine significantly increased after LDN (Fig. 2). The preoperative kidney function did not differ between the groups. However, the median serum creatinine significantly differed postoperatively (RLDN 1.09 vs. LLDN $1.25 \mathrm{mg} / \mathrm{dL}, p=$ $0.045)$.

Over a median follow-up of 45 months (range 6-79 months), the graft function only differed one week postop between right and left kidneys (serum creatinine RLDN 2.1 vs. LLDN $1.72 \mathrm{mg} / \mathrm{dL}, p=0.003$, Fig. 2). Thereafter, the graft function was comparable and did not decrease.
Neither the patient nor the death-censored graft survival significantly differed (Table 3). The 1-, 3-, and 5-year patient survival was 99\%, 97\%, and 95\% for LLDN and $100 \%, 98 \%$, and $98 \%$ for RLDN, correspondingly ( $p=$ $0.747)$. The 1-, 3-, and 5-year death-censored graft survival was $100 \%, 95 \%$, and $89 \%$ for LLDN, and $100 \%, 92 \%$ and $92 \%$ for RLDN, correspondingly ( $p=0.969$ ). Six (3.1\%) patients died during follow-up, 2 from cardiovascular reasons, 2 from sepsis, and 2 from cancer. In total, $9(20.9 \%)$ recipients of right grafts and $28(18.7 \%)$ recipients of left grafts had graft rejections, 9 (4.7\%) grafts were lost during follow-up. The 5-year patient survival was 
95\% for left and 98\% for right grafts, the 5-year deathcensored graft survival $89 \%$ and $92 \%$, correspondingly (Table 3). Both the patient and death-censored graft survival did not differ between RLDN and LLDN (Fig. 3).

\section{Discussion/Conclusion}

This single center study analyzed the outcomes of 204 pure laparoscopic donor nephrectomies and 193 corresponding open KTs within 5 years, and the results were stratified by the side of LDN. At a median caseload of 34 LDNs, 78.4\% LDNs were left-sided. Larger LDN cohorts have been published to date, but their results are often impacted by learning curves or merge different surgical approaches [16-18]. The first LDN at our department has been performed in 1999, wherefore the impact of learning within this cohort is negligible, especially because only experienced surgeons were involved [11]. The surgeons had performed at least 30 laparoscopic tumor nephrectomies before and assisted various LDNs; to guarantee a maximum of safety, younger LDN surgeons were always assisted by experienced ones [19]. Of note, this is the largest cohort of pure LDNs in Germany and the technique has not changed throughout the period [20,21]. Overall, the right and left LDNs did not have different complication rates, but recipients of right grafts had more postoperative complications. However, the long-term follow-up was not impacted, as neither the graft function, nor patient or death-censored graft survival differed between the groups in the mid-term and long term.

As living kidney donors have an above-average health, the complication rates of a donor nephrectomy have to be as low as possible. For both LLDNs and RLDNs, intraoperative complications were rare. Regardless, two RLDNs, but no LLDN, had to be converted to open surgery due to bleedings. Khalil et al. [6] also reported on higher conversion rates for RLDN, but their results were based on donor nephrectomies between 2000 and 2009, the early beginnings of LDN. In contrast, other authors did not find any differences and Wang et al. [7] even stated that LLDN had more intraoperative complications [16, 22, 23]. Importantly, the low intraoperative complication rates of our study translated into low postoperative complication rates for the donors. No RLDN, but 3 (1.5\%) LLDNs were followed by major postoperative complications, which is comparable with others $[6,7]$.

Thereof, RLDN was 19 min shorter than LLDN. Wang et al. [7] also found a difference of $13.4 \mathrm{~min}$ of operating time in their meta-analysis, and according to Abrahams et al. [24], RLDN is shorter and easier. From our experience, RLDN is neither easier nor more challenging than LLDN, but the more complex topology of the left hemiabdomen harboring the spleen could be a reason for the shorter operating times of RLDN. Although the WIT was significantly longer for RLDN, the difference of $25 \mathrm{~s}$ is only marginal from a clinical point of view and has surely not impacted the postoperative graft function. Accordingly, neither Liu et al. [25] nor Wang et al. [7] found differences regarding the WIT in their meta-analyses.

For the open KTs, the intraoperative complication rates did not differ, but the postoperative complication rates within 30 days were higher for kidney recipients of right grafts. In contrast, the complication rates neither differed when stratifying for the implantation side (ipsilateral implantation $50 \%$ vs. contralateral $32.5 \%, p=$ 0.095). Correspondingly, the side of LDN was the only factor in the multiple regression analysis with an impact on postoperative complications of the recipient. Wang et al. [7] did not find any differences in postoperative complication rates in their meta-analysis, but many included works did not apply the Clavien-Dindo classification [3, 6]. In our analysis, 4 out of 5 vascular complications occurred in RLDN group. Accordingly, others reported on more transplant losses due to vascular complications or a tendency toward more vein thromboses with right grafts $[3,26]$. In order to prevent vascular complications and to optimize the outcomes after living KTs, the selection of the graft's side clearly is a crucial aspect. At our institution, this individual case-by-case decision is based on vessel anatomy and split-renal function. Interestingly, there was a higher proportion of right grafts with multiple veins, and there was a tendency toward more left grafts with multiple arteries, in line with others [27, 28]. We do not prefer left over right grafts in case of multiple arteries, and vice versa for multiple veins, but rather choose the kidney with less vessels and the lower split-renal function. As long as the difference of the split-renal function between the right and left kidney does not exceed 5\%, the kidney with the less complex vessel anatomy is chosen, otherwise the kidney with the lower split graft function. We cannot deny a certain reluctance against right grafts in our analysis, as they were chosen less often and their split-renal function was significantly lower. However, most meta-analyses today show that only $20-30 \%$ of LDNs are right-sided and there are still high-volume centers which practically do not to perform RLDNs at all [7, 29].

As a secondary outcome, we compared the kidney and graft function at a median follow-up of 45 months.
Zeuschner et al. 
At discharge, all donors had a worse kidney function, but interestingly it was better for right donors. As neither the preoperative kidney function nor the donor baseline characteristics differed, which are comparable with most other studies, this difference is most likely related to the lower preoperative scintigraphic function of right grafts $[7,25]$. Correspondingly, the median serum creatinine of kidney recipients 1 week after transplantation was $0.4 \mathrm{mg} / \mathrm{dL}$ higher for recipients of right grafts. As the recipient characteristics did neither differ, this can also be related to the lower split function of right grafts, and a tendency towards worse preoperative kidney function of their recipients. Moreover, the DGF rates were higher for right grafts, but this difference was not statistically significant. In this regard, it is unlikely that the significant difference of $11 \mathrm{~min}$ in operating time for the transplantations caused the higher DGF rates because other studies also report on comparable results [30]. Furthermore, the anastomosis times did not significantly differ, although some authors found them to be longer for right grafts. They explained this finding not only with the shorter vein of right grafts but also higher rates of multiple vessels and the relatively long renal artery which tends to an arterial kinking $[6,31]$. The CIT in our study was longer than in some other studies because we do not perform LDN and transplantation simultaneously, but sequentially [22]. However, it was not different between right and left grafts, wherefore one reason for the slightly higher DGF rates of right grafts could be the higher rates of vascular problems. Of note, the graft function was no longer different between right and left grafts during follow-up. Moreover, the death-censored graft and patient survival were excellent and did neither differ between the groups. In contrast, Khalil et al. [6] found a significantly worse graft survival with right grafts and performed a follow-up almost twice as long. We assume that there will not be such differences in our single-center cohort with significant prior expertise in LDN, but we cannot prove this (yet) due to our shorter follow-up.

This retrospective, single center analysis is not devoid of limitations. As we did not perform a prospective, randomized study, our results are impacted by a selection bias. However, the groups did neither differ regarding the donor nor recipient, but only the graft characteristics, which did not appear to impact the long-term follow-up. There have also been analyses of larger cohorts, but these results are often impacted by inherent learning curves of the surgeons, center-specific differences, or different surgical approaches.

Comparison of Right versus Left

Laparoscopic Donor Nephrectomies
To conclude, our large single-center cohort of pure laparoscopic donor nephrectomies revealed differences in terms of the perioperative outcomes regarding the LDN itself and the corresponding KTs. These differences did not impact the long-term follow-up, which is of utmost importance. However, the higher complication rates for the recipients of right kidneys indicate that even experienced transplant surgeons at high-volume centers are challenged by the different anatomical conditions, wherefore RLDN should either be trained or performed more often.

\section{Statement of Ethics}

This study has been conducted according to the Declaration of Helsinki; all patients gave their written informed consent. The article is exempt from the local Ethical Committee approval (Institutional Review Board of Charité Hospital Berlin), and the need for informed consent was waived because of this study's retrospective, noninterventional design and because patient data confidentiality and privacy were always maintained.

\section{Conflict of Interest Statement}

The authors have no conflicts of interest to declare.

\section{Funding Sources}

This study has not received any funding.

\section{Author Contributions}

Conceptualization: L.H. and F.F.; methodology: L.H. and P.Z.; data acquisition: L.H.; analysis: P.Z., L.H., and F.F.; investigation and data curation: P.Z.; writing - original draft preparation: P.Z.; supervision: F.F.; all authors read, revised an approved the manuscript.

\section{References}

1 Breda A, Budde A, Figueiredo A, Lledó García E, Olsburgh J, Regele $\mathrm{H}$, et al. EAU guidelines on renal transplantation; 2020.

2 Broudeur L, Karam G, Chelghaf I, De Vergie S, Rigaud J, Perrouin Verbe MA, et al. Feasibility and safety of laparoscopic living donor nephrectomy in case of right kidney and multiple-renal artery kidney: a systematic review of the literature. World J Urol. 2020;38(4): 919-27.

3 Buell JF, Edye M, Johnson M, Li C, Koffron A, Cho E, et al. Are concerns over right laparoscopic donor nephrectomy unwarranted? Ann Surg. 2001;233(5):645-51. 
4 Liu N, Wazir R, Wang J, Wang K-J. Maximizing the donor pool: left versus right laparoscopic live donor nephrectomy: systematic review and meta-analysis. Int Urol Nephrol. 2014;46:1511-9.

5 Abrahams HM, Meng MV, Freise CE, Stoller ML. Pure laparoscopic right donor nephrectomy: step-by-step approach. J Endourol. 2004;18(3):221-5.

6 Khalil A, Mujtaba MA, Taber TE, Yaqub MS, Goggins W, Powelson J, et al. Trends and outcomes in right vs. left living donor nephrectomy: an analysis of the OPTN/UNOS database of donor and recipient outcomes - should we be doing more right-sided nephrectomies? Clin Transplant. 2015;30:145-53.

7 Wang K, Zhang P, Xu X, Fan M. Right versus left laparoscopic living-donor nephrectomy: a meta-analysis. Exp Clin Transplant. 2015;13: 214-26.

8 Turk IA, Giessing M, Deger S, Davis JW, Fabrizio MD, Schönberger B, et al. Laparoscopic live donor right nephrectomy: a new technique with preservation of vascular length. Transplant Proc. 2003;35(2):838-40.

9 Garcia-Ochoa C, Feldman LS, Nguan C, Monroy-Cuadros M, Arnold J, Boudville N, et al. Donor nephrectomy outcomes research $\mathrm{N}$ : perioperative complications during living donor nephrectomy: results from a multicenter cohort study. Can J Kidney Health Dis. 2019; 6:2054358119857718.

10 Keller JE, Dolce CJ, Griffin D, Heniford BT, Kercher KW. Maximizing the donor pool: use of right kidneys and kidneys with multiple arteries for live donor transplantation. Surg Endosc. 2009;23(10):2327-31.

11 Friedersdorff F, Kothmann L, Manus P, Roigas J, Kempkensteffen C, Magheli A, et al. Long-term donor outcomes after pure laparoscopic versus open living donor nephrectomy: focus on pregnancy rates, hypertension and quality of life. Urol Int. 2016;97(4):450-6.

12 Turk IA, Deger S, Davis JW, Giesing M, Fabrizio MD, Schönberger B, et al. Laparoscopic live donor right nephrectomy: a new technique with preservation of vascular length. J Urol. 2002;167(2 Pt 1):630-3.
13 Giessing M, Deger S, Schönberger B, Türk I, Loening SA. Laparoscopic living donor nephrectomy: from alternative to standard procedure. Transplant Proc. 2003;35(6):2093-5.

14 Zeuschner P, Hennig L, Peters R, Saar M, Linxweiler J, Siemer S, et al. Robot-assisted versus laparoscopic donor nephrectomy: a comparison of 250 cases. J Clin Med. 2020; 9(6):1610.

15 Giessing M, Deger S, Ebeling V, Schönberger B, Roigas J, Kroencke TJ, et al. [Laparoscopic living donor nephrectomy of kidneys with multiple renal vessels]. Urologe A. 2003; 42(2):225-32.

16 Breda A, Veale J, Liao J, Schulam PG. Complications of laparoscopic living donor nephrectomy and their management: the UCLA experience. Urology. 2007;69(1):49-52.

17 Kocak B, Baker TB, Koffron AJ, Leventhal JR. Laparoscopic living donor nephrectomy: a single-center sequential experience comparing hand-assisted versus standard technique. Urology. 2007;70(6):1060-3.

18 Melcher ML, Carter JT, Posselt A, Duh QY, Stoller M, Freise CE, et al. More Than 500 consecutive laparoscopic donor nephrectomies without conversion or repeated surgery. Arch Surg. 2005;140(9):835.

19 Friedersdorff F, Werthemann P, Cash H, Kempkensteffen C, Magheli A, Hinz S, et al. Outcomes after laparoscopic living donor nephrectomy: comparison of two laparoscopic surgeons with different levels of expertise. BJU Int. 2013;111(1):95-100.

20 Wahba R, Kleinert R, Hellmich M, Heiermann N, Dieplinger G, Schlößer HA, et al. Optimizing a living kidney donation program: transition to hand-assisted retroperitoneoscopic living donor nephrectomy and introduction of a passive polarizing three-dimensional display system. Surg Endosc. 2017; 31(6):2577-85.

21 Weigand K, Kawan F, Schumann A, Mohammed N, Lindner F, Fornara P. Rechts- oder linksseitige Donornephrektomie - ist das wirklich relevant? Urologe. 2020;59(1):32-9.

22 Simforoosh N, Tabibi A, Soltani MH, Zare S, Yahyazadeh SR, Abadpoor B. Long-term follow-up after right laparoscopic donor nephrectomy and inverted kidney transplant. Exp Clin Transplant. 2016;14:27-31.
23 Tsoulfas G, Agorastou P, Ko D, Hertl M, Elias $\mathrm{N}$, Cosimi $\mathrm{AB}$, et al. Laparoscopic living donor nephrectomy: is there a difference between using a left or a right kidney? Transplant Proc. 2012;44(9):2706-8.

24 Abrahams HM, Freise CE, Kang SM, Stoller ML, Meng MV. Technique, indications and outcomes of pure laparoscopic right donor nephrectomy. J Urol. 2004;171(5):1793-6.

25 Liu N, Wazir R, Wang J, Wang KJ. Maximizing the donor pool: left versus right laparoscopic live donor nephrectomy: systematic review and meta-analysis. Int Urol Nephrol. 2014;46(8):1511-9.

26 Nunes-Carneiro D, Marques-Pinto A, Veiga C, Braga I, Cabral JF, Almeida M, et al. Which one is the best for living donation: a multipleartery left kidney nephrectomy or a right kidney nephrectomy? Transplant Proc. 2019; 51(5):1559-62.

27 Engelken F, Friedersdorff F, Fuller TF, Magheli A, Budde K, Halleck F, et al. Pre-operative assessment of living renal transplant donors with state-of-the-art imaging modalities: computed tomography angiography versus magnetic resonance angiography in $118 \mathrm{pa}-$ tients. World J Urol. 2013;31(4):983-90.

28 Kapoor A, Lambe S, Kling AL, Piercey KR, Whelan PJ. Outcomes of laparoscopic donor nephrectomy in the presence of multiple renal arteries. Urol Ann. 2011;3(2):62-5.

29 Ozturk SA, Yuksel Y, Erbis H, Aliosmanoglu I, Sarier M, Yayar O, et al. Laparoscopic live donor nephrectomy: experience of high-volume center with 2,477 cases. Urol Int. 2021; 105(1-2):100-7.

30 Simforoosh N, Basiri A, Tabibi A, Shakhssa$\lim$ N, Hosseini Moghaddam SM. Comparison of laparoscopic and open donor nephrectomy: a randomized controlled trial. BJU Int. 2005;95(6):851-5.

31 Hellegering J, Visser J, Kloke HJ, D’Ancona FC, Hoitsma AJ, van der Vliet JA, et al. Deleterious influence of prolonged warm ischemia in living donor kidney transplantation. Transplant Proc. 2012;44(5):1222-6. 